\title{
Compact Planar L-band Antenna Arrays with Optimal Diversity Performance
}

\author{
S. Irteza ${ }^{1}$, N. Murtaza $^{2}$, S. Caizzone ${ }^{3}$, R. Stephan ${ }^{4}$, and M. A. Hein ${ }^{5}$
}

\begin{abstract}
At L-band (1-2GHz) due to significantly large freespace wavelengths, compact antenna arrays with small interelement separation i.e., $d<\lambda / 2$, are a suitable choice for overall size reduction. However, mutual coupling becoming prominent in compact arrays results in a degradation of the diversity degreesof-freedom, which are required for beamforming capabilities in modern receivers. In this paper we discuss a potential approach to mitigate this limitation. We present empirical results for an eigenmode analysis applied to the radiation matrices of compact planar arrays, derived from the far-field integration of complex realised-gain matrices. Furthermore, optimal arrangements for compact planar arrays with respect to the highest possible value of minimum eigenmode efficiency are discussed. It could be shown that planar arrays have higher efficiencies and lower radiation correlation, hence better diversity degrees-of-freedom than linear arrays, particularly in a compact configuration.
\end{abstract}

\section{INTRODUCTION}

Recent advances in radio frequency (RF) CMOS technology have led to compact low-noise receiver designs [1], [2]. However, overall antenna array size in these modern miniaturised receivers has been mostly limited to a separation between neighbouring elements equal to $d=\lambda / 2$, thus limiting the overall compactness of the system. Especially, in case of L-band where $\lambda \approx 20 \mathrm{~cm}$, this large separation among elements becomes a limiting factor for designing a compact array. A wide-spread application in L-band is global satellite navigation, where multi-element antenna arrays are becoming attractive for accurate and robust reception of the navigation signal in presence of interferers. As a result, in addition to compactness, this application demands efficient arrays with maximal diversity capability and low-noise receivers.

Compact arrays with an inter-element separation $d<$ $\lambda / 2$ severely suffer from mutual coupling [3], hence degrade the overall performance of the system. Digital beamforming at baseband could mitigate the effects of coupling for a compact array to some extent, though at the expense of reduced dynamic range or radiative degrees-of-freedom [4], [5]. In the recent past, techniques for reducing coupling have been reported, using decoupling and matching networks [6] involving eigenmode excitation, or defected ground structures as an electromagnetic bandgap configuration in printed antennas [7]. In conjunction with addressing coupling reduction between elements, it is necessary to optimise the array design with respect to

\footnotetext{
$1,2,4,5$ Institute for Information Technology, RF and Microwave Research Lab, Ilmenau University of Technology, Germany.

${ }^{3}$ Institute of Communications and Navigation, Germany Aerospace Center (DLR), Wessling,Germany.

1 email: safwat-irteza.butt@tu-ilmenau.de, tel: +49-3677-691361.
}
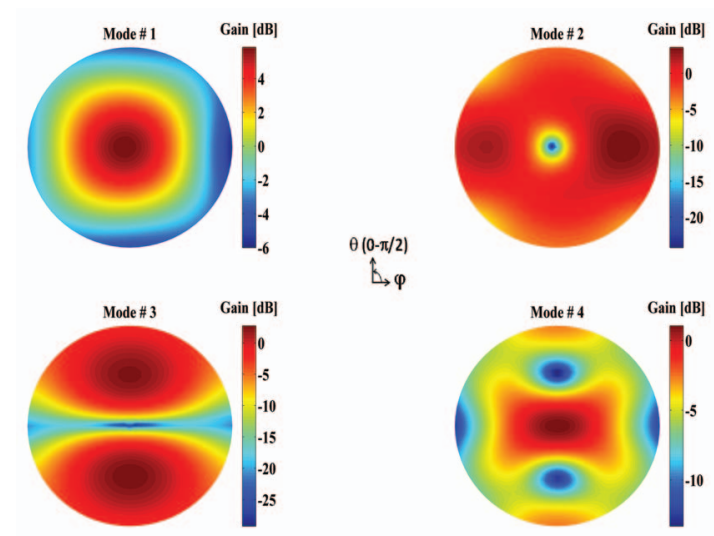

Fig. 1: Eigenmode gain patterns for a 4-element planar lossless array in square arrangement with $d=\lambda / 4$, where mode\# 1 has in-phase $(++++)$ excitation for elements, mode $\# 2$ has $(--++)$ excitation with one null, mode\#3 has $(-++-)$ excitation with two nulls, mode\#4 has $(+-+-)$ excitation with three nulls. mode\#1 has the maximum gain, and mode\#4 has the minimum gain but maximum degrees-of-freedoms.

its diversity degrees-of-freedom. Several methods involving, e.g., an analysis of the superdirectivity sensitivity factor, mutual impedance, or active reflection coefficient have been identified [8]-[10] but lack complete scalable performance characterisation. In [6], an approach was introduced which utilises eigenmode radiation efficiencies to analyse the performance of a compact array. In this paper, we extend this approach to the computation of the radiation matrix using realised-gain matrices, making it applicable for both lossy and lossless arrays, which is used to derive the eigenmodes. These eigenmodes, as illustrated in Fig. 1 for a 4-element array, form orthogonal sub-space vectors, where the correlation $\rho_{i j}=0$ between any two basis vectors, making them independent and hence perfectly decoupled. In general, eigenmode patterns indicate the highest-order eigenmode (mode\#4 in Fig. 1) with $n-1$ nulls to be least efficient. Therefore, keeping the minimal modal radiation efficiency or eigenmode efficiency $\left(\lambda_{\min }\right)$ as high as possible may provide a valuable optimisation tool for the design of compact arrays.

In Section II, we present the design of a compact single radiator and chosen arrangements for an $n$-element array. In Section III, we present the extended analysis of compact arrays. In Section IV, we investigate the figure-of-merit of compact arrays by simulations. Optimised planar arrays for maximal diversity performance are discussed in Section V. In the end, conclusions are given based on the simulated results in Section IV. 


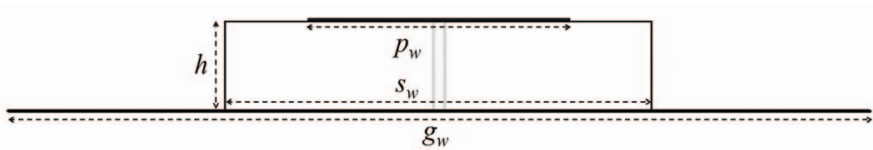

Fig. 2: Single element design - square patch with reduced substrate size.

\section{Design of Compact Arrays}

\subsection{Radiating Element}

The compactness of an antenna array is limited mainly by the inter-element separation $d$, and the size of the individual radiation elements. Therefore, initially a miniaturised single radiator element is considered. We have designed a linearly polarised square patch on a high-ielectric permittivity substrate ECCOSTOCK HiK 500F with $\epsilon_{r}=30$ [11], where $\lambda_{g}=$ $0.18 \lambda$, and an almost $80 \%$ reduced size in comparison to free-space wavelength. In order to increase the impedance bandwidth, a substrate thickness of $h=6.4 \mathrm{~mm}$ was chosen. The antenna design sketched in Fig. 2 is optimised at the L-band frequency $1.575 \mathrm{GHz}$ for maximum impedance bandwidth and radiation efficiency, which results in a ground length $g_{l}=48 \mathrm{~mm}$, and a patch length $p_{l}=14.86 \mathrm{~mm}$. We also considered a reduced substrate over the ground plane, where the substrate width $s_{w}$ is only $24 \mathrm{~mm}$, to minimise the dielectric losses. This small dielectric size has no obvious advantage for coupling reduction, as the ground plane dimension remains large.

\subsection{Array Configurations}

Different array configurations with different numbers of elements were modelled and simulated in CST Microwave Studio [12]. Planar arrays represent 2-D geometries as compared to 1-D geometries of linear arrays. These arrays were optimised with respect to inter-element separations, and geometry configurations for maximal diversity degrees-of-freedom, as mentioned before and discussed further in later sections. Different geometries for $n=3,4,6$, and 9 elements were designed with inter-element separations $d$ as shown in Fig. 3 .

\section{Analysis of Compact Arrays}

In order to evaluate an antenna array, it is desired to model and analyse the parameters which encompass the effects of power dissipated, i.e., lost within the antenna, and reflected due to impedance mismatch, along with power radiated in the presence of coupling between neighbouring elements. Power dissipation within the antenna is mainly due to the losses in the dielectric substrate materials and to the finite conductivity of metal surfaces. Therefore, their practical characterisation in performance analysis is necessary, especially in the case of printed antennas. Compact arrays inherit a finite real part of mutual impedances $Z_{i j}$, which result in feed impedances for individual radiators different from their self impedances for beamforming, giving rise to reflection losses. All these effects negatively affect the total efficiency of the antenna. Therefore,

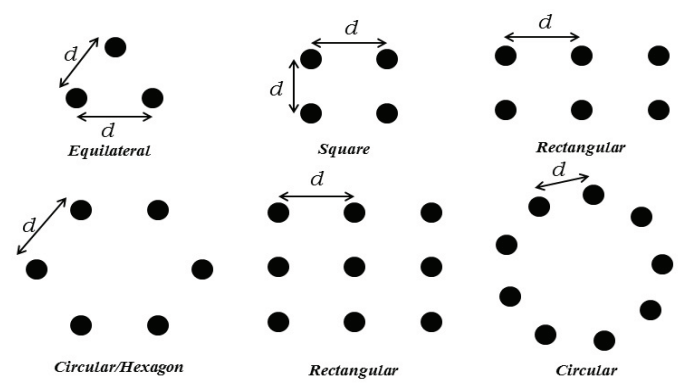

Fig. 3: Array geometry configurations for $n$-elements investigated in this work.

our main concern has been to formulate an algorithm for obtaining the radiation matrix $\tilde{H}$ of the designed array, and then determine the realised radiation efficiencies including all above mentioned losses. In [6], C. Volmer et al. have introduced a technique for deriving the radiation matrix from the scattering matrix $S_{i j}$, assuming a lossless antenna array:

$$
\begin{gathered}
P_{r a d}=P_{i n}-P_{a v g}=\vec{a}^{H} \vec{a}-\vec{b}^{H} \vec{b} \\
=\vec{a}^{H}\left(\vec{I}-\vec{S}^{H} \vec{S}\right) \vec{a} \\
\tilde{H}_{a c c}=\vec{I}-\vec{S}^{H} \vec{S},
\end{gathered}
$$

whereas we use the complex valued realised-gain matrices of the antenna array as compared to isotropic radiators $G_{i}(\theta, \phi)$ for determining the radiation matrix [13]:

$$
\begin{gathered}
\tilde{H}_{\text {rad }_{\theta}}=\frac{1}{4 \pi} \oint G_{i_{\theta}}(\theta, \phi) \cdot G_{j_{\theta}}(\theta, \phi) d \Omega \\
\tilde{H}_{\text {rad }_{\phi}}=\frac{1}{4 \pi} \oint G_{i_{\phi}}(\theta, \phi) \cdot G_{j_{\phi}}(\theta, \phi) d \Omega \\
\tilde{H}_{r a d}=\tilde{H}_{\text {rad }_{\theta}}+\tilde{H}_{r_{a d}}
\end{gathered}
$$

Here, $\tilde{H}_{\text {rad }}$ includes the losses dissipated within the antenna array, and hence, represents the true radiated power. It may be noted that in case of a lossless array $\tilde{H}_{\text {rad }}=\tilde{H}_{a c c}$.

\subsection{Eigen-Analysis}

The radiation matrix $\tilde{H}$ can be diagonalised to obtain the eigenvectors and eigenvalues. The eigenvectors represent the excitation vectors for a multi-element array providing the decoupled excitations and the respective eigenvalues (eigen-efficiencies or modal radiation efficiencies). Each eigen-efficiency represents the power of the respective eigenmode actually radiated into the far-field [6]:

$$
\begin{gathered}
\tilde{H}=\tilde{Q} \Lambda \tilde{Q}^{H} \\
\text { where } \Lambda=\operatorname{diag}\left\{\lambda_{1}, \lambda_{2}, \ldots \lambda_{n}\right\}
\end{gathered}
$$

Therefore, the worst-case radiation efficiency will be given by $\lambda_{\min }=\min \left\{\vec{\lambda}_{i}\right\}$. 


\subsection{Radiation Pattern Correlation Analysis}

In presence of mutual coupling, the diagonal elements of $\tilde{H}_{\text {rad }}$ represent the amount of power radiated by the individual elements, the off-diagonal elements are a measure of correlation/overlap between the $i$ and $j$ elements. Therefore, a correlation coefficient for mutually coupled compact arrays can be represented as [14]:

$$
\rho_{i j}=\frac{\tilde{H}_{i j}}{\sqrt{\tilde{H}_{i i} \tilde{H}_{j j}}}
$$

where $\rho_{i j}$ is a complex number with absolute value of 1 for full beam overlap and 0 for orthogonal beams. In literature, this correlation coefficient $\tilde{\rho}$ is often treated as a figure-of-merit for compact arrays.

\section{Figure-of-Merit for Compact Arrays}

\subsection{Correlation coefficient $\rho$}

If we consider an array with $n=4$ and $d=\lambda / 4$ in a square configuration, the radiation matrix computed from eq. (5) is given as

$$
\tilde{H}_{\text {rad }}=\left[\begin{array}{cccc}
0.58 & 0.19 & 0.01 & 0.09 \\
0.17 & 0.57 & 0.07 & 0 \\
0.13 & 0.07 & 0.56 & 0.18 \\
0.09 & 0 & 0.18 & 0.58
\end{array}\right]
$$

and, using eq. (8), the correlation matrix is given as:

$$
\rho_{s q}=\left[\begin{array}{cccc}
1 & 0.32 & 0.03 & 0.16 \\
0.33 & 1 & 0.13 & 0.01 \\
0.03 & 0.13 & 1 & 0.32 \\
0.16 & 0.01 & 0.32 & 1
\end{array}\right] .
$$

However, in contrast to the square arrangement, for the linear array the correlation matrix is:

$$
\rho_{\text {lin }}=\left[\begin{array}{cccc}
1 & 0.34 & 0.30 & 0.05 \\
0.34 & 1 & 0.51 & 0.29 \\
0.33 & 0.10 & 1 & 0.36 \\
0.05 & 0.29 & 0.36 & 1
\end{array}\right]
$$

It is observed by comparison, that a linear array has an average correlation $\rho_{\text {avg }}=\frac{1}{n(n-1)}\left(\sum_{i j, i \neq j} \rho_{i j}\right)$ of $31 \%$ in contrast to a much lower value of $16 \%$ for the planar array, which indicates the superior diversity performance of a planar array for a given number of elements and inter-element separation. However, eigenvector excitation will lead to $\rho_{a v g}=0$, giving perfect decorrelation, orthogonality, and decoupling of the radiation patterns, thus making the correlation coefficient meaningless for the comparison of different compact array configurations. Therefore, we discuss an additional figure-of-merit based on eigen-analysis.

\subsection{Worst-case eigen-efficiency $\lambda_{\min }$}

The diversity loss $L_{d}$ of mutually coupled antenna arrays with respect to its ideal uncoupled counterpart as defined in [15] can be equated in terms of eigen-efficiencies as:

$$
L_{d} \approx-\frac{10}{n} \log \left[\prod_{i=1}^{n} \lambda_{i}\right] \quad d B
$$

TABLE I: Diversity loss and minimum, maximum, average modal radiation eigen-efficiencies in $\%$ for $n=4$ and $d=\lambda / 4$ and $\lambda / 5$ in linear, square and circular configurations.

\begin{tabular}{|c|c|c|c|c|}
\hline & \multicolumn{3}{|c|}{$d=\lambda / 4$} & $d=\lambda / 5$ \\
\hline Modes & linear & square & circular & square \\
\hline \hline 1 & 88 & 83 & 83 & 71 \\
2 & 81 & 68 & 78 & 40 \\
3 & 66 & 48 & 39 & 30 \\
4 & 2 & 29 & 9 & 14 \\
\hline$\lambda_{\min }$ & 2 & 29 & 9 & 14 \\
\hline$\lambda_{\text {avg }}$ & 77 & 57 & 53 & 38 \\
\hline$\lambda_{\max }$ & 88 & 83 & 83 & 71 \\
\hline $\mathbf{L}_{\mathbf{d}_{\mathbf{d B}}}$ & $\mathbf{5 . 0 2}$ & $\mathbf{2 . 7 6}$ & $\mathbf{4 . 0 2}$ & $\mathbf{4 . 8 1}$ \\
\hline
\end{tabular}

In Table I, planar and linear lossless arrays for $n=4$ for $d=\lambda / 4$ and $\lambda / 5$ are compared in terms of $\lambda_{\text {min }}$, average eigen-efficiency $\lambda_{\text {avg }}=\frac{1}{n} \sum_{i=1}^{n} \lambda_{i}$, maximum eigen-efficiency $\lambda_{\max }$, and $L_{d}$. It becomes evident that $\lambda_{\text {avg }}$ and $\lambda_{\max }$ are optimistic parameters for analysing the diversity performance of compact arrays in contrast to $\lambda_{m i n}$. The lower the minimum eigen-efficiency, the higher becomes the diversity loss. For example, for an array with $n=4$ and $d=\lambda / 4$, it is shown in Table I that $\lambda_{\min }=2 \%$ in the linear configuration, while for the planar array it improves dramatically to $29 \%$. In contrast, comparing $\lambda_{\text {avg }}, \lambda_{\max }=77 \%, 88 \%$ and $57 \%, 83 \%$ respectively, suggests that the linear configuration were better than planar. The results for the diversity loss clearly indicate a gain of $2.26 \mathrm{~dB}$ for the planar arrangement, which is an implication of its higher $\lambda_{\text {min }}$. We conclude that an optimal diversity performance can be achieved by improving the $\lambda_{\text {min }}$-values of compact arrays.

\section{Optimisation of Compact Planar arrays}

\subsection{Optimal Geometry}

Now, we wish to optimise an $n=6$ element lossless planar array, with respect to $\lambda_{\min }$, by altering the arrangement of the individual radiating elements. We choose two inter-element separations as shown in Table II, $d=\lambda / 2$ where coupling is minimal, and $d=\lambda / 4$ having prominent coupling effects. It can be noticed that for the case of minimal coupling, an improvement of $\lambda_{\min }$ is not possible by using different geometrical arrangements. However, for $d=\lambda / 4$, the optimal arrangement turned out to be circular or hexagonal, where both provide a minimum eigenefficiency of $21 \%$, in contrast to merely $6 \%$ for a rectangular arrangement. Therefore, it can be generalised that a $n$-element compact array, where coupling is prominent, can be optimised in geometry with respect to maximum $\lambda_{\text {min }}$.

\subsection{Optimal Inter-Element Separation $d$}

The design of compact arrays requires reduced inter-element separations. However, for a given number of $n$ elements and reduced separation, $\lambda_{\min }$ becomes very low, e.g., for $n=4$ and $d=\lambda / 5$ in planar configuration its value drops to 
TABLE II: $\lambda_{\min }$ in $\%$, different geometry configurations for $n=6$ and $d=\lambda / 2$ and $\lambda / 4$.

\begin{tabular}{|c|c|c|c|}
\hline \multirow{2}{*}{ Optimal Geometry } & Shape & $\begin{array}{c}n=6 \\
d=\lambda / 2\end{array}$ & $\begin{array}{c}n=6 \\
d=\lambda / 4\end{array}$ \\
\hline \hline Linear & & 62 & 2 \\
\hline Planar & Rectangular & 64 & 6 \\
\cline { 2 - 4 } & Circular/Hexagonal & 64 & 21 \\
\hline
\end{tabular}

$14 \%$ as shown in Fig. 4. Therefore, for the $n$-element array, to maintain all degrees-of-freedom efficient, thus above a threshold value of $\lambda_{\min }$, a compromise has to be found for the choice of $d$, limiting the overall compactness. If we consider the simulated results of Fig. $4, n=6$ and $d=\lambda / 4$ in circular/hexagonal configuration is a suitable choice with sufficiently high $\lambda_{\min }$ and diversity performance.

\section{Conclusions}

We have considered compact planar arrays for applications at L-band frequencies, such as robust satellite navigation. Compactness causes mutual coupling between neighbouring radiating elements, which makes the array inefficient. As the introduction of beamforming capability is required in compact arrays, direction dependent reflection losses occur as a further consequence. Therefore, a complete eigen-analysis using eigenmode excitations encompassing both mutual coupling and mismatch effects has been considered. The analysis has shown that a correlation coefficient for distinguishing mutually coupled arrays is of limited use, especially when the excitations are orthogonal, e.g., eigenvector excitations. Rather, the minimal eigen-efficiency $\lambda_{\min }$ has been identified as a suitable optimisation parameter for compact arrays. It has also been shown that planar arrays display higher $\lambda_{\text {min }}$ values compared to linear arrangements, and accordingly provide better diversity performance. Furthermore, planar array geometries can be optimised for $\lambda_{\min }$, i.e., in case of $n=6$ a circular arrangement is preferred over rectangular, whereas for $n=4$, a square arrangement performs better than circular. It is worth-mentioning that these results have been inferred from simulations, and practical implementations of such systems may result in different quantitative values, as manufacturing tolerances may give rise to smaller eigen-efficiency values. Therefore, we are on the way to implement such systems, and evaluate the resulting diversity performance.

\section{ACKNOWLEDGMENT}

The authors would like to thank Dr. C. Volmer for his valuable suggestions and able guidance during this work. This work was supported by the German Aerospace Center (DLR) on behalf of the German Federal Ministry of Economics and Technology under Grant 50NA1007.

\section{REFERENCES}

[1] T. Y. Chin, S. F. Chang, J.C. Wu, and C. C. Chang, A 25-GHz Compact Low-Power Phased-Array Receiver With Continuous Beam Steering in CMOS Technology, IEEE Journal of Solid-State Circuits, vol. 45, no. 11, November 2010

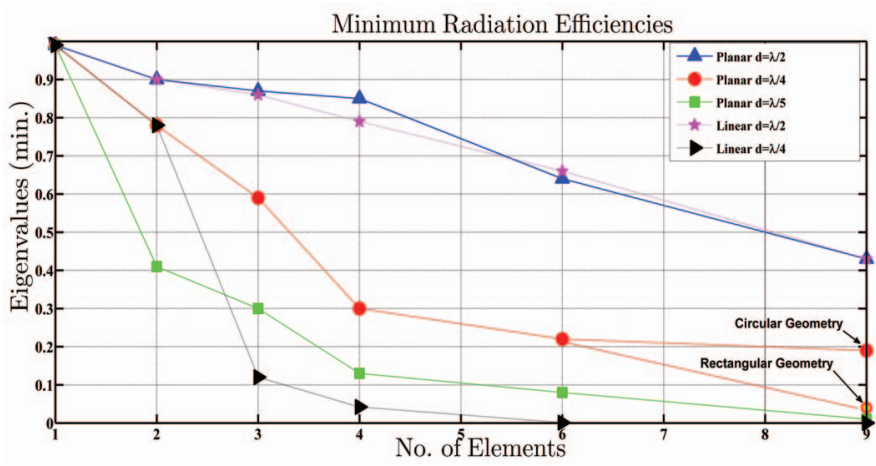

Fig. 4: Minimum eigen-efficiency for lossless arrays versus number of radiating elements, for different inter-element separations and geometrical arrangements as indicated in the figure.

[2] J. Ferreira et al. LNA, Oscillator and Mixer Co-Design for Compact RFCMOS ISM Receivers, 16th International Conference on Mixed Design of Integrated Circuits and Systems, June 25-27, 2009, Lodz, Poland

[3] I.J. Gupta and A.A. Ksienski, Effect of Mutual Coupling on the Performance of Adaptive Arrays, IEEE Trans. Antennas and Propogation, vol. 31 pp. 785-791, 1983

[4] R.G. Vaughan and J.B Anderson, Antenna diversity in mobile communications, IEEE Trans. Veh. Technol. Vol. 36 pp. 149-172, 1987

[5] H. J. Chaloupka and X. Wang, Novel approach for diversity and MIMO antennas at small mobile platforms, in Proceeding of 15th IEEE International Symposium on Personal Indoor and Mobile Radio Communications (PIMRC 04), 2004

[6] C. Volmer, J. Weber, R. Stephan, K. Blau, M. A. Hein, An Eigen-Analysis of Compact Antenna Arrays and Its Application to Port Decoupling, IEEE Transactions on Antennas and Propagation, vol. 56, no. 2, pp.360-370 February, 2008

[7] Qian Li, A.P. Feresidis, Reduction of Mutual Coupling between Compact MIMO Antenna Arrays, IEEE Antennas and Propagation Conference 8-9 November 2010, Loughborough, UK

[8] E. N. Gilbert and S. P. Morgan, Optimum design of directive antenna arrays subject to random variations, Bell Syst. Tech. J., vol. no. 34, pp.637663, May 1955.

[9] A. C. Ludwig, Mutual coupling, gain, and directivity of an array of two identical antennas, IEEE Trans. Antennas Propag., vol.no. 6, pp. 837841, Nov. 1976.

[10] S. Drabowitch, A. Papiernik, H. D. Grifths, J. Encinas, and B. L. Smith, Modern Antennas, 2nd ed. Dordrecht, The Netherlands:Springer, 2005

[11] Y. Zhou, C.C. Chen and J.L. Volakis, Single-fed Circularly Polarised Antenna Element With Reduced Coupling for GPS Arrays, IEEE Transactions on Antennas and Propagation, vol. 56, no.5, pp. 1469-1472 May 2008

[12] CST Microwave Studio 2011, http//www.cst.com

[13] C. Volmer, Compact antenna arrays in mobile communications: A quantitative analysis of radiator coupling Ilmenau: Univ.-Verl. Ilmenau, 2010. - XV, 250 p. ISBN 978-3-939473-71-8

[14] A. Stjernman, Relationship between radiation pattern correlation and scattering matrix of lossless and lossy antennas, Electronic Letters, vol. 41, no. 12 , pp. 678-680, June 2005

[15] C. Volmer, J. Weber, R. Stephan, and M. A. Hein, A descriptive model for analyzing the diversity performance of compact antenna arrays, IEEE Transacations on Antennas and Propagation, vol. 57, no. 2, February 2009 